\title{
Native Electrospray and Electron-Capture Dissociation in FTICR Mass Spectrometry Provide Top-Down Sequencing of a Protein Component in an Intact Protein Assembly
}

\author{
Hao Zhang, Weidong Cui, Jianzhong Wen, Robert E. Blankenship, and \\ Michael L. Gross \\ Department of Chemistry, Washington University, St. Louis, Missouri, USA
}

\begin{abstract}
The intact yeast alcohol dehydrogenase (ADH) tetramer of $147 \mathrm{kDa}$ was introduced into a FTICR mass spectrometer by native electrospray. Electron capture dissociation of the entire $23+$ to $27+$ charge state distribution produced the expected charge-reduced ions and, more unexpectedly, 39 c-type peptide fragments that identified $\mathrm{N}$-terminus acetylation and the first 55 amino acids. The results are in accord with the crystal structure of yeast $\mathrm{ADH}$, which shows that the C-terminus is buried at the assembly interface, whereas the $\mathrm{N}$-terminus is exposed, allowing ECD to occur. This remarkable observation shows promise that a top-down approach for intact protein assemblies will be effective for characterizing their components, inferring their interfaces, and obtaining both proteomics and structural biology information in one experiment. (J Am Soc Mass Spectrom 2010, 21, 1966-1968) (C) 2010 American Society for Mass Spectrometry
\end{abstract}

$\mathrm{M}$ ass spectrometry (MS) is evolving as an important approach for investigating intact, large protein assemblies in the gas phase [1], augmenting other approaches [2]. Advances in quadrupole time-of-flight instruments and ion mobility have underpinned this new role in structural biology [3-5]. Determinations of molecular weight, stoichiometry, and assembly patterns of essential biological assemblies as large as MDa hepatitis B virus assemblies are now possible [6].

To identify the constituents of a protein complex, information about the subunits of a complex is needed. Collisionally activated dissociation (CAD) [1], blackbody infrared dissociation (BIRD) [7], electron-capture dissociation (ECD) [8], infrared multiphoton dissociation (IRMPD) [9], and surface-induced dissociation (SID) [10] can yield some sequence information for proteins in large assemblies. For example, multiple collisions at $\mathrm{keV}$ laboratory energy demonstrate that native protein complexes can be disassembled into peptide fragments [11]. ECD [12], now an established tool for top-down sequencing and for determining post-translational modifications, can successfully characterize, in part, proteins of MW up to $200 \mathrm{kDa}$ [13] and noncovalent protein-ligand complexes [14]. Applications of ECD to protein-protein assemblies [8], however, are sparse.

Address reprint requests to Professor. M. L. Gross, Department of Chemistry, Washington University in St. Louis, One Brookings Drive, St. Louis, MO 63130, USA. E-mail: mgross@wustl.edu
ECD and FT (Fourier transform) ion cyclotron resonance (ICR) MS combine as an appealing approach for study of protein assemblies because, in principle, the masses of the complex and of the subunits, as well as some sequence of the constituents can be acquired in a single experiment. Indeed, we report here a successful ECD-based FTICR MS top-down approach that affords the composition, stoichiometry, and partial sequence of a $147 \mathrm{kDa}$ noncovalent protein assembly; namely, the yeast alcohol dehydrogenase (ADH) tetramer.

\section{Experimental}

Fresh yeast alcohol dehydrogenase (ADH) complex (Sigma, St. Louis, MO, USA) $(1.7 \mu \mathrm{M})$ was prepared by buffer exchange before every experiment and sprayed from aqueous $\mathrm{NH}_{4} \mathrm{OAc}(1 \mathrm{M})$. Custom spray tips were pulled from Polymicro silicon tubing (360 $\mu$ m o.d., 150 $\mu \mathrm{m}$ i.d., Phoenix, AZ, USA) by using a microcapillary puller (Sutter Instrument Co., Novato, CA, USA). The sample solution was infused at $25-100 \mathrm{~nL} / \mathrm{min}$ (Harvard PHD Ultra syringe pump, Instech Laboratories, Inc., Plymouth Meeting, PA, USA). A Bruker SolariX 12 T FTICR mass spectrometer (Bruker Daltonics, Bremen, Germany) with capabilities for CAD, ETD, ECD, and sustained off-resonance irradiation (SORI) was used for analysis.

\section{Results and Discussion}

We successfully sprayed (native ESI) the yeast alcohol dehydrogenase (ADH) assembly, obtaining significantly lower charged proteins $(23+$ to $27+$, Figure 1a) 


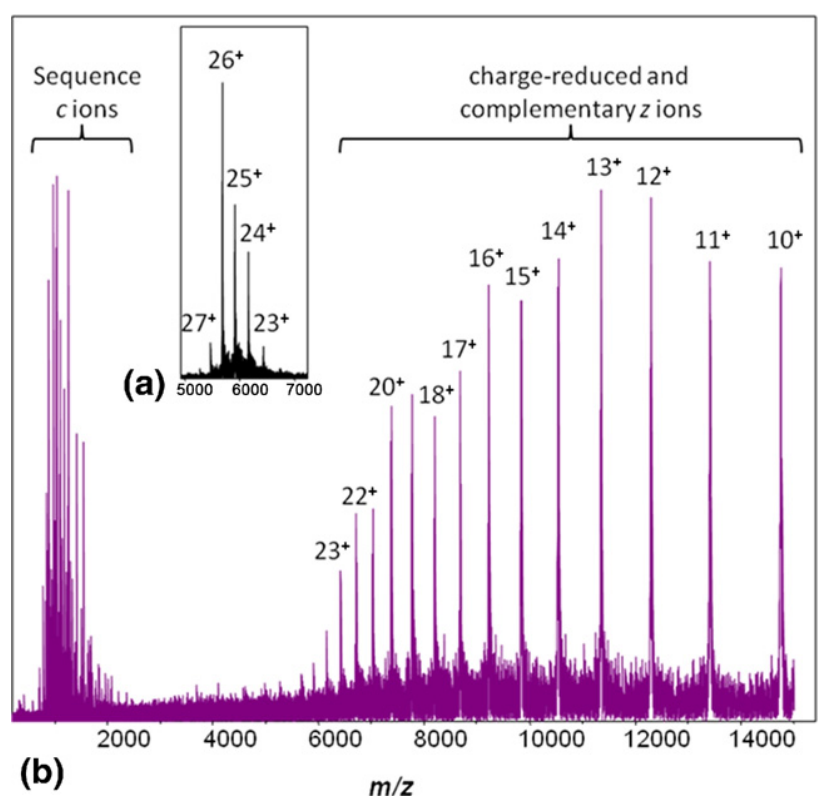

Figure 1. (a) Mass spectrum and (b) ECD product-ion spectrum of the yeast $\mathrm{ADH}$ complex.

compared with what would be observed by non-native ESI. Charge deconvolution gave a MW of $147.5 \mathrm{kDa}$, verifying that we had introduced the tetramer.

We conducted collisional activation in the front end of the hybrid instrument (before the FTICR trap) and found it to be inadequate to fragment the complex, suggesting tight binding of the protein constituents. We also were unable to detect any fragments by using electron-transfer dissociation (ETD). Given the large $\mathrm{m} / \mathrm{z}$ difference of the complex and ETD reagent ion, the precursor ions may not have optimally situated for the ETD reaction. We considered front-end selection by the quadrupole mass analyzer, but this is not yet possible on this instrument for ions in this $\mathrm{m} / \mathrm{z}$ range. When we attempted in-trap isolation and ECD of the most abundant $26+$ charge state, only charge reduction was observed owing to poor dynamic range. The observation matches that of Geels et al. [8] on ECD of the 84 kDa gp31 heptamer; only charge reduction and no peptide fragments were produced. The high MW ion packet isolated inside the ICR trap was likely displaced from the trap center owing to perturbations from the waveforms used for isolation. This led to poorer overlap between the electron beam for ECD and the stored ion packet than when activating lower $m / z$ ions [15].

When we submitted the entire, narrow charge-state distribution to ECD, without isolation, we obtained a remarkable result (Figure 1b) whereby the precursor ions were completely depleted, and two types of product ions formed: (1) a set of peptide fragments of $m / z<$ 2000, and (2) charge-reduced precursors down to at least $10+$, accompanied presumably by high $\mathrm{m} / \mathrm{z}$ ions that are complements to the peptide fragments. To our knowledge, this is the first observation of peptide fragments produced directly by ECD from a protein constituent of a protein assembly.
Analysis of the low $\mathrm{m} / \mathrm{z}$ pattern shows that the first residue is acetylated serine, not methionine. Moreover, 39 c-type ions up to the $55^{\text {th }}$ residue form in the fragmentation from the $\mathrm{N}$ terminus (Figure 2). Missing in the pattern are ions from chain cleavages at Pro23, 25, 27 , and 55 , which cannot be seen because there is no mass change when the 5-membered ring is cleaved to give a c-type ion. Nevertheless, those fragments that are produced are sufficient to identify the protein (see below).

Moreover, the fragments are consistent with the X-ray crystal structure (Figure 3 ) that shows the $\mathrm{N}$ terminus is free and available for fragmentation whereas the $C$ terminus is buried at the interface. In this case, the transition to the gas phase preserves sufficient solid-state structure to enable these phenomena. This complex is a dimer of dimers [16], held together by nearly a score of salt bridges [17]. The ionic forces that maintain protein higher order structure and hold together assemblies of this nature become stronger in the gas phase, suggesting why ECD affords sequence information rather than disrupts the assembly. It also explains why we can sequence through the first 55 residues of the $\mathrm{N}$ terminus, which is not involved in the interface. Further, the complementary z-ions are likely seen accompanying each of the charge-reduced species at slightly lower $m / z$, dispersed by their complex isotope patterns.

One means to characterize large protein complexes by MS is a "bottom-up" strategy whereby the proteins are denatured leading to release of the subunits, the components separated and proteolyzed, or simply proteolyzed, and LC/tandem MS and database searching are applied to identify the constituents. More refinement comes from high-performance QTOF MS of macromolecular assemblies, revealing stoichiometry and connectivity of subunits [4]. Our observation suggests that a top-down approach for characterization of pro-

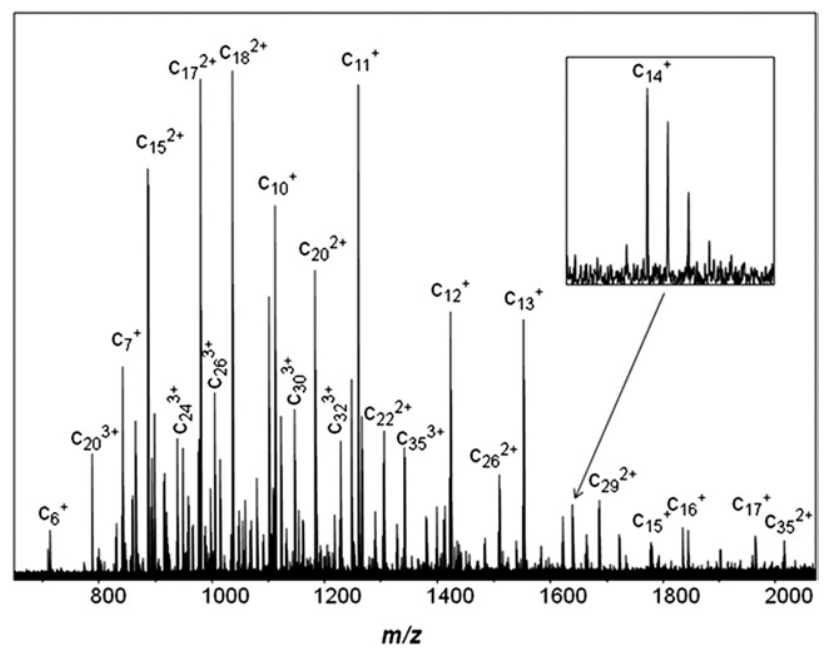

Figure 2. ECD mass spectrum of the ADH complex over the low $\mathrm{m} / \mathrm{z}$ range of Figure 1. 
ac I PE TQKGV I FYESHGKLEYKD I PVPKPKANE LL INVKYSGVCH TDLHAWHGDWPLPVKL PLVGGHE GAGVVVGMGENVKGWKI GDYA GIKWLNGS CMACEYCELGNESNCPHADL SGYTHDGSFQEYATAD AVQAAHIPQGTDLAEVAPVLCAGITVYKALKSANLMAGHWVAIS GAAGGL GS LAVQYAKAMGYRVLG IDGGE GKEEL FRS IGGEVF ID FTKEKDIVGAVLKATDGGAHGVINVSVSEAAIEASTRYVRANGT TVLVGMPAGAKCC SDVFNQVVKS ISIVGSYVGNRADTREALDF F ARGLIKSP IKVVGLSTLPE IYEKMEKGQ IVGRYVVDTSK

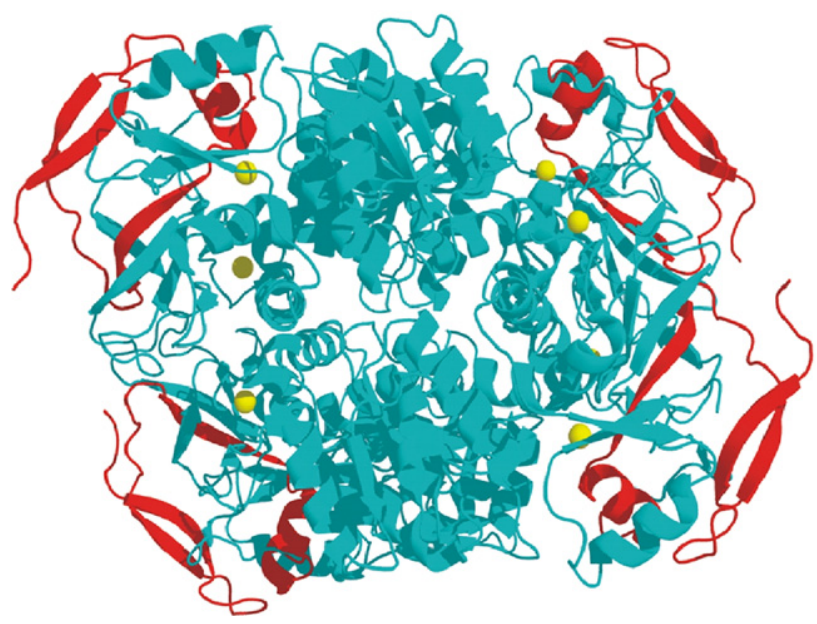

Figure 3. ADH sequence showing the sites of ECD cleavages (top) and crystal structure of the yeast ADH tetramer (2HCY in PDB) with the $\mathrm{N}$-terminal 55 residues that were sequenced highlighted in red (bottom).

tein complexes directly by tandem mass spectrometry will be successful.

Assuming the constituents of this complex are unknown and that top-down sequencing can be successful, we tested whether the sequence from ECD was sufficient to identify the protein. Although there are a number of ways to accomplish this, we chose to generate sequence tags [18] (7-20 amino acid) by using Bruker Biotools and extended them with consideration of the proline gaps. We then submitted the three longest tags to Mascot searching against the NCBI database with a mass tolerance of $0.02 \mathrm{Da}$ to identify the published Chain A, yeast alcohol dehydrogenase I (gi 112491285) containing 347 residues and having MW of $36.7 \mathrm{kDa}$. The Mascot peptide score was 294 (expectation value $1.5 \mathrm{e}-16)$. Given that the measured molecular weight of the complex is $147.5 \mathrm{kDa}$, it is straightforward to conclude that four $\mathrm{ADH}$ monomers make up the whole assembly.

In conclusion, this approach using FTICR MS applied to the yeast ADH assembly afforded the MW of the complex. More importantly, ECD generated sufficient sequence information to identify reliably the constituent protein. Given the long run of ECD fragments, one can pinpoint that region of the protein that is relatively free in the $\mathrm{ADH}$ tetramer, and likely to be remote from the interface of this gas-phase assembly.

More generally, this "top-down" approach may offer a more efficient and simpler procedure than a "bottomup" approach for characterization of each constituent in a macromolecular assembly. Furthermore, as a one-step protocol, it demonstrates the integration of MS-based proteomics and structural biology in one platform and satisfies a sought-after goal [19]. In future experiments, we will focus on methodology, namely development of front-end preselection of precursor ions for ECD and addition of IRMPD to the platform for better fragmentation efficiency. We are also studying other homogeneous and ultimately heterogeneous assemblies, the results of which will be the subject of future publications.

\section{Acknowledgments}

The authors acknowledge research funding from the National Center for Research Resource of the NIH (grant P41RR000954), and instrumentation funding from the High-End Instrument Program of the NCRR (grant 1 S10 025101).

\section{References}

1. Benesch, J. L.; Ruotolo, B. T.; Simmons, D. A.; Robinson, C. V. Protein Complexes in the Gas Phase: Technology for Structural Genomics and Proteomics. Chem. Rev. 2007, 107(8), 3544-3567.

2. Robinson, C. V.; Sali, A.; Baumeister, W. The Molecular Sociology of the Cell. Nature 2007, 450, 973-982.

3. Benesch, J. L. Collisional Activation of Protein Complexes: Picking up the Pieces. J. Am. Soc. Mass Spectrom. 2009, 20, 341-348.

4. Sharon, M. How Far Can We Go with Structural Mass Spectrometry of Protein Complexes? J. Am. Soc. Mass Spectrom. 2010, 21, 487-500.

5. van Duijn, E. Current Limitations in Native Mass Spectrometry Based Structural Biology. J. Am. Soc. Mass Spectrom. 2010, 21, 971-978.

6. Uetrecht, C.; Versluis, C.; Watts, N. R.; Roos, W. H.; Wuite, G. J.; Wingfield, P. T.; Steven, A. C.; Heck, A. J. High-Resolution Mass Spectrometry of Viral Assemblies: Molecular Composition and Stability of Dimorphic Hepatitis B Virus Capsids. Proc. Natl. Acad. Sci. U.S.A. 2008, 105, 9216-9220.

7. Felitsyn, N.; Kitova, E. N.; Klassen, J. S. Thermal Decomposition of a Gaseous Multiprotein Complex Studied by Blackbody Infrared Radiative Dissociation. Investigating the Origin of the Asymmetric Dissociation Behavior. Anal. Chem. 2001, 73, 4647-4661.

8. Geels, R. B.; van der Vies, S. M.; Heck, A. J.; Heeren, R. M. Electron Capture Dissociation as Structural Probe for Noncovalent Gas-Phase Protein Assemblies. Anal. Chem. 2006, 78, 7191-7196.

9. El-Faramawy, A.; Guo, Y.; Verkerk, U.; Thomson, B. A.; Siu, M. Evaluation of IR Multi photon Dissociation as a Method for High Mass Protein Clean up. Proceedings of the 56th ASMS Conference on Mass Spectrometry, Denver, CO, 2008.

10. Jones, C. M.; Beardsley, R. L.; Galhena, A. S.; Dagan, S.; Cheng, G.; Wysocki, V. H. Symmetrical Gas-Phase Dissociation of Noncovalent Protein Complexes Via Surface Collisions. J. Am. Chem. Soc. 2006, 128, 15044-15045.

11. Benesch, J. L.; Ruotolo, B. T.; Sobott, F.; Wildgoose, J.; Gilbert, A.; Bateman, R.; Robinson, C. V. Quadrupole-Time-of-Flight Mass Spectrometer Modified for Higher-Energy Dissociation Reduces Protein Assemblies to Peptide Fragments. Anal. Chem. 2009, 81, 1270-1274.

12. Zubarev, R. A.; Kelleher, N. L.; McLafferty, F. W. Electron Capture Dissociation of Multiply Charged Protein Cations. A Nonergodic Process. J. Am. Chem. Soc. 1998, 120, 3265-3266.

13. Han, X.: Jin, M. Breuker, K.; McLafferty, F. W. Extending Top-Down Mass Spectrometry to Proteins with Masses Greater than 200 Kilodaltons. Science 2006, 314, 109-112.

14. Xie, Y.; Zhang, J.; Yin, S.; Loo, J. A. Top-Down ESI-ECD-FT-ICR Mass Spectrometry Localizes Noncovalent Protein-Ligand Binding Sites. J. Am. Chem. Soc. 2006, 128(45), 14432-14433.

15. Guan, S.; Burlingame, A. L. High Mass Selectivity for Top-Down Proteomics by Application of SWIFT Technology. J. Am. Soc. Mass Spectrom. 2010, 21(3), 455-459.

16. Powers, E. T.; Powers, D. L. A Perspective on Mechanisms of Protein Tetramer Formation. Biophys. J. 2003, 85, 3587-3599.

17. Casadio, R.; Martelli, P. L.; Giordano, A.; Rossi, M.; Raia, C. A. A Low-Resolution 3D Model of the Tetrameric Alcohol Dehydrogenase from Sulfolobus Solfataricus. Protein Eng. 2002, 15(3), 215-223.

18. Mortz, E.; O'Connor, C.; P. B.; Roepstorff, P.; Kelleher, N. L.; Wood, T. D.; McLafferty, F. W.; Mann, M. Sequence Tag Identification of Intact Proteins by Matching Tandem Mass Spectral Data Against Sequence Data Bases. Proc. Natl. Acad. Sci. U.S.A. 1996, 93(16), 8264-8267.

19. Zhou, M.; Robinson, C. V. When Proteomics Meets Structural Biology. Trends Biochem Sci. doi:10.1016/j.tibs. 2010.04.007. 